\title{
Ácido indolbutírico e substratos na alporquia de umbuzeiro ${ }^{1}$
}

\author{
Tiago Reis Dutra ${ }^{2}$, Marília Dutra Massad ${ }^{3}$, Mateus Felipe Quintino Sarmento², Jéssica Costa de Oliveira ${ }^{2}$
}

\begin{abstract}
Indolebutyric acid and

substrates on Spondias tuberosa layering

Spondias tuberosa is a native species from the Brazilian Northeast and northern Minas Gerais State, whose fruits present good market acceptance. However, its large scale propagation is affected by slow and uneven seeds germination, demanding the use of vegetative propagation techniques. This study aimed at evaluating the effect of indolebutyric acid (IBA) application and different kinds of substrate on the vegetative propagation of Spondias tuberosa by layering. The experiment consisted of eleven Spondias tuberosa arrays and a randomized blocks design with four replications, in a $3 \times 5$ factorial scheme, in order to evaluate the effect of five IBA doses $\left(0 \mathrm{mg} \mathrm{L}^{-1} ; 1,000 \mathrm{mg} \mathrm{L}^{-1}\right.$; 2,000 $\mathrm{mg} \mathrm{L}^{-1} ; 4,000 \mathrm{mg} \mathrm{L}^{-1}$; and 6,000 $\left.\mathrm{mg} \mathrm{L}^{-1}\right)$ and three substrates (Bioplant $^{\mathbb{R}}$, vermiculite and sugarcane bagasse). The selected branches were ringed and, subsequently, the concentrated IBA solutions were applied. At 180 days, the percentage of rooted and callous layers, percentage of layers with primordium roots, number of roots per layer, length of the longest root and root fresh and dry weight were evaluated. The use of $6,000 \mathrm{mg} \mathrm{L}^{-1}$ of IBA promoted a higher growth and development of radicular layers. The use of sugarcane bagasse is recommended, for being easy to get, in the study area, besides presenting adequate chemical and physical features.
\end{abstract}

KEY-WORDS: Spondias tuberosa Arr. Câmara; vegetative propagation; rooting; sugarcane bagasse.

\section{INTRODUÇÃO}

O Semiárido brasileiro possui grande riqueza florestal. Encontram-se, nesta região, as maiores ocorrências de plantas xerófitas do mundo, além de uma grande variedade de plantas endêmicas (Campos 2010).

O umbuzeiro (Spondias tuberosa Arr. Cam.) pertence à família Anacardiaceae. A espécie carac-

\section{RESUMO}

O umbuzeiro é uma espécie nativa do Nordeste brasileiro e norte do Estado de Minas Gerais, cujos frutos apresentam grande aceitação no mercado. Entretanto, a propagação em larga escala da espécie é dificultada pela lenta e desuniforme germinação das sementes, exigindo o uso de técnicas de propagação vegetativa. $\mathrm{O}$ presente trabalho objetivou avaliar o efeito da aplicação de ácido indolbutírico (AIB) e diferentes tipos de substratos na propagação vegetativa de umbuzeiro, via técnica de alporquia. O experimento foi desenvolvido utilizando-se onze matrizes de Spondias tuberosa (umbuzeiro) e delineamento de blocos casualizados, com quatro repetições, em esquema fatorial $5 \times 3$, sendo avaliados o efeito de cinco doses de AIB $\left(0 \mathrm{mg} \mathrm{L}^{-1}\right.$, $1.000 \mathrm{mg} \mathrm{L}^{-1}, 2.000 \mathrm{mg} \mathrm{L}^{-1}, 4.000 \mathrm{mg} \mathrm{L}^{-1}$ e $6.000 \mathrm{mg} \mathrm{L}^{-1}$ ) e três tipos de substratos (Bioplant ${ }^{\mathbb{R}}$, vermiculita e bagaço de cana). Os ramos selecionados foram anelados e, posteriormente, aplicadas as soluções concentradas de AIB. Aos 180 dias, foram mensurados a percentagem de alporques enraizados, com calo e com primórdios radiculares, número de raízes por alporque, comprimento da maior raiz e massa fresca e seca das raízes. A utilização de $6.000 \mathrm{mg} \mathrm{L}^{-1}$ de AIB promoveu maior crescimento e desenvolvimento do sistema radicular dos alporques. O uso do bagaço de cana é recomendado, por se tratar de insumo de fácil obtenção, na região em estudo, além de apresentar características químicas e físicas adequadas.

PALAVRAS-CHAVE: Spondias tuberosa Arr. Câmara; propagação vegetativa; enraizamento; bagaço de cana.

teriza-se como uma planta xerófita e caducifólia, originária das zonas menos chuvosas do Nordeste brasileiro e norte do Estado de Minas Gerais (Neves et al. 2007). A espécie produz frutos com boa aparência e qualidade nutritiva, muito consumidos "in natura”, mas com grande potencial para utilização na forma processada, como polpa congelada, sucos e sorvetes (Lima et al. 2002). Os frutos apresentam apelo "exótico", para mercados de outras regiões do

1. Trabalho recebido em ago./2012 e aceito para publicação em nov./2012 (nº registro: PAT 19657).

2. Instituto Federal de Educação, Ciência e Tecnologia do Norte de Minas Gerais (IFNMG), Campus Salinas, Salinas, MG, Brasil.E-mails: tiagoreisdutra@gmail.com,mateusengflorestal@hotmail.com,jessicataiocosta2010@hotmail.com.

3. Instituto Federal de Educação, Ciência e Tecnologia do Paraná (IFPR), Campus Irati, Irati, PR, Brasil.

E-mail: mariliamassad@yahoo.com.br. 
Brasil, como o Sudeste e o Sul, bem como para o mercado externo, o que vem estimulando o aumento na sua produção (Folegatti et al. 2003).

Um dos grandes entraves ao cultivo comercial de espécies frutíferas nativas do Semiárido é a obtenção de mudas com a qualidade desejada. Segundo Souza et al. (2005), uma das dificuldades na propagação do umbuzeiro, em larga escala, é a dormência das sementes, a qual propicia emergência lenta e desuniforme de plântulas.

Outro problema para o estabelecimento de plantios comerciais da espécie é a produção tardia de plantas propagadas seminalmente, sendo o uso de técnicas de propagação vegetativa, visando, principalmente, à antecipação do período reprodutivo, uma alternativa para a exploração econômica do umbuzeiro.

Dentre os principais métodos de propagação vegetativa, destaca-se a alporquia, considerada o mais simples e menos drástico processo de propagação vegetativa, e que requer menor cuidado com o ambiente (Campos 2010).

A alporquia pode ser utilizada em diversas plantas que apresentem dificuldades de multiplicação e que tenham ramos lenhosos ou semilenhosos. O sucesso desta técnica pode ser potencializado, por meio da correta escolha e utilização de alguns insumos, destacando-se o tipo de substrato e o uso de reguladores de crescimento.

O substrato precisa fornecer água, oxigênio e nutrientes (Lima et al. 2010), permitindo o crescimento das raízes e, assim, fornecendo suporte estrutural à parte aérea dos alporques e garantindo o desenvolvimento de uma planta de qualidade. Para isto, deve-se considerar suas características físicas e químicas, como homogeneidade, baixa densidade, alta porosidade, boa capacidade de retenção de água, alta capacidade de troca catiônica e nutrientes em quantidades suficientes para o bom desenvolvimento das mudas (Gomes \& Silva 2004, Dutra et al. 2012b).

Além do substrato, o processo de formação de raízes em alporques pode estar relacionado, também, com o uso de reguladores de crescimento, sendo a aplicação exógena de auxinas sintéticas uma das formas mais estudadas no favorecimento do balanço hormonal, para o enraizamento e uniformidade do sistema radicial.

Dentre as auxinas utilizadas, o ácido indolbutírico (AIB) vem se destacando como o mais comumente empregado na indução do enraizamento adventício, por se tratar de uma substância com maior fotoestabilidade, em comparação com as demais auxinas sintéticas, além de apresentar ação localizada e menor sensibilidade à degradação biológica (Smarsi et al. 2008).

A aplicação de AIB tem sido utilizada na produção de mudas de umbuzeiro, entretanto, vem sendo mais empregada para as técnicas de enxertia e estaquia (Lima 2009) e superação de dormência de sementes (Melo et al. 2012), necessitando-se de estudos sobre sua utilização, para a formação de alporques da espécie.

Diante do exposto, objetivou-se, com o presente trabalho, avaliar o efeito da aplicação de concentrações de AIB e diferentes tipos de substratos na propagação vegetativa de umbuzeiro, utilizando-se a técnica de alporquia.

\section{MATERIAL E MÉTODOS}

O trabalho foi desenvolvido utilizando-se onze matrizes de Spondias tuberosa (umbuzeiro) com oito anos de idade, provenientes de propagação vegetativa por enxertia, localizadas na Fazenda Santa Izabel, pertencente ao Instituto Federal de Educação, Ciência e Tecnologia do Norte de Minas Gerais (IFNMG), Campus Salinas, de abril a outubro de 2011.

$\mathrm{O}$ estudo foi conduzido em delineamento de blocos casualizados, com quatro repetições, em esquema fatorial $5 \times 3$, sendo os fatores constituídos por cinco doses de AIB (0 mg L-1, $1.000 \mathrm{mg} \mathrm{L}^{-1}$, $2.000 \mathrm{mg} \mathrm{L}^{-1}, 4.000 \mathrm{mg} \mathrm{L}^{-1}$ e $\left.6000 \mathrm{mg} \mathrm{L}^{-1}\right)$ e três tipos de substrato (Bioplant ${ }^{\circledR}$, vermiculita de granulometria média e bagaço de cana peneirado em malha de 4,0 mm), onde cada unidade experimental foi constituída por seis alporques.

Os alporques foram realizados na parte mediana de ramos de boa sanidade e vigor, com diâmetro entre $1,0 \mathrm{~cm}$ e 2,0 cm. A quantidade de alporques por árvore variou entre 15 e 25 , sendo determinada de acordo com a disponibilidade de ramos que possuíam as características necessárias para o desenvolvimento da técnica.

Os ramos selecionados foram anelados, com o uso de um estilete comum, removendo-se, completamente, a casca em sua volta, formando um anel com 1,5 cm de largura. No local dos anelamentos, ocorreram aplicações de cerca de $0,5 \mathrm{~mL}$ das soluções concentradas de AIB, sobre toda a extensão do ferimento, com o auxílio de pincel. O preparo das so- 
luções concentradas foi feito diluindo-se $1,0 \mathrm{~g} ; 2,0 \mathrm{~g}$; 4,0 g e 6,0 g de AIB em 1,0 L de solução alcoólica a $50 \%$, isto é, $50 \%$ de álcool absoluto e $50 \%$ de água destilada, obtendo-se as concentrações desejadas. Em seguida, foi colocado sobre o anel um saco de polietileno $(25,00 \mathrm{~cm} \times 35,00 \mathrm{~cm} \times 0,02 \mathrm{~cm})$ contendo os diferentes tipos de substratos, previamente umedecidos. Posteriormente, as extremidades superiores e inferiores dos sacos foram amarradas e receberam uma etiqueta, para a identificação do tratamento.

Foi efetuada a caracterização química dos substratos (Embrapa 1997), bem como avaliadas as características físicas de porosidade total, macroporosidade, microporosidade e capacidade máxima de retenção de água (Carvalho \& Silva 1992) (Tabela 1).

Após 30 dias da realização dos alporques, uma nova aplicação de água $(25,0 \mathrm{~mL})$ foi realizada, em cada tratamento, com o auxílio de uma seringa, procedimento que se repetiu a cada 15 dias, após a primeira reposição de água, até o final do experimento.

Aos 180 dias após a realização dos alporques, os ramos foram removidos das plantas matrizes, com o auxílio de uma tesoura de poda. Em seguida, os sacos plásticos foram retirados, para eliminação do substrato presente nos mesmos, procedendo-se à

Tabela 1. Características químicas e físicas dos substratos utilizados no enraizamento de alporques de umbuzeiro (Salinas, MG, 2011).

\begin{tabular}{|c|c|c|c|}
\hline \multirow[b]{2}{*}{ Características* } & \multicolumn{3}{|c|}{ Substrato } \\
\hline & Bioplant $^{\circledR}$ & Vermiculita & $\begin{array}{l}\text { Bagaço } \\
\text { de cana }\end{array}$ \\
\hline pH (água) & 5,00 & 6,00 & 5,50 \\
\hline M.O. $\left(\right.$ dag $\left.\mathrm{kg}^{-1}\right)$ & 7,90 & 2,72 & 6,30 \\
\hline $\mathrm{P}\left(\mathrm{mg} \mathrm{dm}^{-3}\right)$ & 776,48 & 76,84 & 7,30 \\
\hline $\mathrm{K}\left(\mathrm{mg} \mathrm{dm}^{-3}\right)$ & $1.410,00$ & 467,00 & 60,80 \\
\hline $\mathrm{Ca}\left(\mathrm{cmol}_{\mathrm{c}} \mathrm{dm}^{-3}\right)$ & 12,22 & 5,29 & 0,40 \\
\hline $\operatorname{Mg}\left(\mathrm{cmol}_{\mathrm{c}} \mathrm{dm}^{-3}\right)$ & 4,72 & 9,54 & 0,24 \\
\hline $\mathrm{H}+\mathrm{Al}\left(\mathrm{cmol}_{\mathrm{c}} \mathrm{dm}^{-3}\right)$ & 8,10 & 1,60 & 1,50 \\
\hline $\mathrm{Al}\left(\mathrm{cmol}_{\mathrm{c}} \mathrm{dm}^{-3}\right)$ & 0,17 & 0,10 & 0,17 \\
\hline $\mathrm{T}\left(\mathrm{cmol}_{\mathrm{c}} \mathrm{dm}^{-3}\right)$ & 20,73 & 15,44 & 0,97 \\
\hline $\mathrm{T}\left(\mathrm{cmol}_{\mathrm{c}} \mathrm{dm}^{-3}\right)$ & 28,66 & 16,94 & 2,30 \\
\hline $\mathrm{SB}\left(\mathrm{cmol}_{\mathrm{c}} \mathrm{dm}^{-3}\right)$ & 20,56 & 15,34 & 0,80 \\
\hline$M(\%)$ & 1,00 & 0,60 & 18,00 \\
\hline $\mathrm{V}(\%)$ & 72,00 & 91,00 & 35,00 \\
\hline Porosidade total $\left(\mathrm{dm}^{3} \mathrm{dm}^{-3}\right)$ & 62,57 & 55,67 & 82,39 \\
\hline Macroporosidade $\left(\mathrm{dm}^{3} \mathrm{dm}^{-3}\right)$ & 19,57 & 17,01 & 40,01 \\
\hline Microporosidade $\left(\mathrm{dm}^{3} \mathrm{dm}^{-3}\right)$ & 43,01 & 38,65 & 42,38 \\
\hline CMRA (mL $\left.55 \mathrm{~cm}^{-3}\right)$ & 23,65 & 21,26 & 23,31 \\
\hline
\end{tabular}

* M.O. = matéria orgânica; $\mathrm{t}=$ capacidade efetiva de troca de cátions; $\mathrm{T}=$ capacidade de troca de cátions; $\mathrm{SB}=$ soma de bases; $\mathrm{m}=$ saturação por alumínio; $\mathrm{V}=$ saturação por bases; $\mathrm{CMRA}$ = capacidade máxima de retenção de água. lavagem em água corrente. Posteriormente, foram avaliados a percentagem de alporques enraizados, com calo e com primórdios radiculares, número de raízes por alporque, comprimento da maior raiz e massa fresca e seca das raízes.

O comprimento das raízes foi medido com o auxílio de uma régua e expresso em centímetros, e a massa fresca da raiz foi obtida pela retirada das raízes com estilete, realizando-se, em seguida, as pesagens do material, em balança de precisão. Para determinação de sua massa seca, todo este material, separado por tratamentos, foi acondicionado em sacos de papel e seco em estufa com circulação forçada de ar (aproximadamente $65^{\circ} \mathrm{C}$ ), até massa constante.

Os dados foram submetidos à análise de variância e, quando o efeito do tipo de substrato foi significativo, as médias foram comparadas pelo teste Tukey $(p<0,05)$. Os efeitos das concentrações de AIB foram analisados por meio de regressões e o valor de $\mathrm{F}$ foi corrigido, sendo apresentadas somente as equações cujos coeficientes de maior grau foram significativos $(\mathrm{p}<0,05)$.

\section{RESULTADOS E DISCUSSÃO}

Não houve efeito significativo da interação tipo de substrato $\mathrm{x}$ doses de AIB para nenhuma das características avaliadas, ocorrendo somente efeitos isolados da concentração do fitohormônio e do substrato (Tabela 2).

O tipo de substrato influenciou apenas na percentagem de primórdios radiculares (Tabela 2). Para esta variável, a vermiculita (35\%) foi capaz de proporcionar índices percentuais 1,8 vezes superior à média dos demais substratos (Tabela 2).

A ausência de efeito do tipo de substrato, para as demais variáveis, demonstra que, nas condições em que foi conduzido o trabalho, o umbuzeiro apresentou grande plasticidade adaptativa, podendo, suas mudas, serem produzidas com qualquer tipo de substrato. Entretanto, o uso do bagaço de cana se torna mais interessante, pois constitui uma alternativa de baixo custo e fácil disponibilidade, já que a cana-de-açúcar é matéria-prima para o principal e mais conhecido produto da região (cachaça artesanal), além de diminuir o acúmulo no meio ambiente.

Além do aspecto econômico, o bagaço de cana apresenta características físicas de porosidade total, macroporosidade e capacidade máxima de retenção de água, bem como valor de $\mathrm{pH}$ e teor de 
Tabela 2. Percentagem de calos, primórdios radiculares e enraizamento de alporques, número de raízes (NRZ), comprimento da maior raiz (COMR), matéria fresca da raiz (MFR) e matéria seca da raiz (MSR) de alporques de umbuzeiro avaliados aos 180 dias após a realização da alporquia, em função do substrato e concentração de ácido indolbutírico (Salinas, MG, 2011).

\begin{tabular}{|c|c|c|c|c|c|c|c|}
\hline \multirow{2}{*}{ Tratamentos } & Calos & Primórdios & Enraizamento & \multirow{2}{*}{ NRZ } & COMR & MFR & MSR \\
\hline & & $\%$ & & & $-\mathrm{cm}-$ & \multicolumn{2}{|c|}{$\mathrm{g}$} \\
\hline \multicolumn{8}{|l|}{ Substrato (S) } \\
\hline Bioplant $t^{\mathbb{B}}$ & 56,11 & $22,22 b$ & $21,67 \mathrm{a}$ & $2,11 \mathrm{a}$ & $4,83 \mathrm{a}$ & $2,66 \mathrm{a}$ & $0,42 \mathrm{a}$ \\
\hline Vermiculita & 41,67 & $35,00 \mathrm{a}$ & $23,33 \mathrm{a}$ & $2,12 \mathrm{a}$ & $2,05 \mathrm{a}$ & $2,88 \mathrm{a}$ & $0,41 \mathrm{a}$ \\
\hline Bagaço de cana & 63,33 & $15,00 \mathrm{c}$ & $21,67 \mathrm{a}$ & $2,12 \mathrm{a}$ & $3,82 \mathrm{a}$ & $1,98 \mathrm{a}$ & $0,33 \mathrm{a}$ \\
\hline \multicolumn{8}{|c|}{ Concentrações $(C)\left(\mathrm{mg} \mathrm{L}^{-1}\right)$} \\
\hline 0 & 61,11 & 25,00 & 13,89 & 0,00 & 0,00 & 0,00 & 0,00 \\
\hline 1.000 & 62,96 & 12,04 & 25,00 & 0,00 & 0,00 & 0,00 & 0,00 \\
\hline 2.000 & 69,44 & 11,11 & 19,44 & 0,00 & 0,00 & 0,00 & 0,00 \\
\hline 4.000 & 30,55 & 44,44 & 25,00 & 0,00 & 0,00 & 0,00 & 0,00 \\
\hline 6.000 & 44,44 & 27,78 & 27,78 & 0,00 & 0,00 & 0,00 & 0,00 \\
\hline Ajuste de regressão & ns & ns & ns & $\mathrm{RL}^{*(1)}$ & $\mathrm{RL}^{* *(2)}$ & $\mathrm{RL}^{*(3)}$ & $\mathrm{RL}^{* *(4)}$ \\
\hline \multicolumn{8}{|l|}{ Teste F (Valor de F) } \\
\hline $\mathrm{S}$ & $1.825,60^{\mathrm{ns}}$ & $1.538,59^{*}$ & $13,89^{\mathrm{ns}}$ & $0,06^{\mathrm{ns}}$ & $11,21^{\mathrm{ns}}$ & $4,90^{\mathrm{ns}}$ & $0,03^{\mathrm{ns}}$ \\
\hline $\mathrm{C}$ & $2.272,40^{\mathrm{ns}}$ & $1.670,54^{\mathrm{ns}}$ & $277,78^{\text {ns }}$ & $1,01 *$ & $22,93 *$ & $31,16^{*}$ & $0,65 * *$ \\
\hline $\mathrm{S} * \mathrm{C}$ & $633,50^{\mathrm{ns}}$ & $149,69^{\text {ns }}$ & $569,44^{\text {ns }}$ & $0,70^{\text {ns }}$ & $10,01^{\mathrm{ns}}$ & $2,41^{\mathrm{ns}}$ & $0,05^{\mathrm{ns}}$ \\
\hline C.V. $(\%)$ & 17,33 & 15,65 & 14,73 & 11,16 & 17,91 & 12,97 & 11,46 \\
\hline
\end{tabular}

Médias seguidas da mesma letra, na coluna, não diferem entre si, pelo teste Tukey, a $5 \%$.

$* *, *$ e ns: Significativo a $1 \%$ e $5 \%$ e não significativo, respectivamente.

${ }^{(1)} \mathrm{y}=1,5215+0,00023 * \mathrm{x}, \mathrm{R}^{2}=0,869 ;{ }^{(2)} \mathrm{y}=-0,1903+0,0013^{* *} \mathrm{x}, \mathrm{R}^{2}=0,972 ;^{(3)} \mathrm{y}=-0,5274+0,001 * * \mathrm{x}, \mathrm{R}^{2}=0,929 ;{ }^{(4)} \mathrm{y}=-0,0722+0,00016^{*} \mathrm{x}, \mathrm{R}^{2}=0,897$

potássio (Tabela 1), classificados como adequados, para substratos utilizados na produção de mudas, segundo classificação proposta por Gonçalves \& Poggiani (1996 apud Dutra et al. 2012a). Desta forma, este substrato apresenta potencial, como meio de crescimento para a produção de mudas de umbu, utilizando-se a técnica de alporquia.

Para as variáveis número de raízes, comprimento da maior raiz e massa fresca e seca de raiz, houve resposta linear crescente às doses de AIB (Tabela 2). Segundo Ohland et al. (2009), dentre as principais funções biológicas das auxinas, pode-se citar o crescimento de órgãos, especialmente as raízes. Comportamento semelhante foi observado por Rios et al. (2012), no enraizamento de estacas de umbu. Souza (2007) constatou não haver efeito das diferentes concentrações deste fitohormônio no comprimento e número de raízes, em alporques da mesma espécie.

Quanto ao percentual de alporques enraizados, não foi observado efeito das concentrações de AIB, o mesmo ocorrendo para os tipos de substrato (Tabela 2). A formação de calos a partir dos 60 dias, característica notada em observações de rotina, durante a condução do trabalho, não se estendeu, na maioria dos casos, para a formação de raízes, uma vez que os mesmos foram avaliados aos 180 dias após a sua confecção, apresentando somente calos, e não raízes. De acordo com Oliveira et al. (2008), isto pode ser explicado pelo fato de as folhas e raízes poderem competir por carboidrato e, neste sentido, o tecido caloso que se formou, provavelmente, foi um dos fatores da não ocorrência de rizogênese.

Nas condições em que foi desenvolvido o trabalho, a aplicação de $6.000 \mathrm{mg} \mathrm{L}^{-1}$ de AIB foi a mais recomendada para esta técnica, devido à sua capacidade de promover crescimento superior de raízes nos alporques, sendo, este resultado, de grande importância, haja vista que a qualidade do sistema radicular reflete diretamente na sobrevivência das mudas em campo, principalmente em épocas menos favoráveis ao desenvolvimento das plantas (Lima et al. 2008, Loss et al. 2009).

Entretanto, segundo alguns autores (Hartmann et al. 2002, Pizzatto et al. 2011), existe um nível ótimo de concentração da auxina, para estimular o máximo crescimento e diferenciação dos tecidos radiculares. Como as respostas para o número e comprimento de raízes e produção de massa fresca e seca do sistema radicular foram linearmente crescentes, com as doses de AIB, ou seja, não foi alcançado o máximo desenvolvimento, sugere-se a realização de futuros estudos, com doses mais elevadas deste fitohormônio, visando a melhorias na técnica de alporquia, para o umbuzeiro. 


\section{CONCLUSÕES}

1. A utilização de AIB promoveu o crescimento do sistema radicular dos alporques de umbuzeiro, sendo a concentração de $6.000 \mathrm{mg} \mathrm{L}^{-1}$, nas condições avaliadas, a mais recomendada para a realização da técnica.

2. As mudas de umbuzeiro podem ser satisfatoriamente produzidas nos três tipos de substrato estudados, comprovando a grande plasticidade adaptativa da espécie, entretanto, o uso do bagaço de cana é o mais recomendado, por se tratar de um insumo de fácil obtenção, na região em estudo, além de apresentar características químicas e físicas adequadas.

\section{AGRADECIMENTOS}

À Fundação de Amparo à Pesquisa do Estado de Minas Gerais (Fapemig), pela concessão de bolsa de iniciação científica.

\section{REFERÊNCIAS}

CAMPOS, G. N. F. Clonagem de Cnidoscolus phyllacanthus (Mart.) Pax et $k$. hoffm. (faveleira) por alporquia. 2010. 57 f. Dissertação (Mestrado em Ciências Florestais) Universidade Federal de Campina Grande, Patos, 2010.

CARVALHO, C. M.; SILVA, C. R. Determinação das propriedades fisicas de substrato. Botucatu: Universidade Estadual Paulista, 1992.

DUTRA, T. R. et al. Desenvolvimento inicial de mudas de copaíba sob diferentes níveis de sombreamento e substratos. Revista Ciência Agronômica, Fortaleza, v. 43, n. 2, p. 321-329, 2012a.

DUTRA, T. R. et al. Emergência e crescimento inicial da canafístula em diferentes substratos e métodos de superação de dormência. Revista Caatinga, Mossoró, v. 25, n. 2 , p. $65-71,2012$ b.

EMPRESA BRASILEIRA DE PESQUISA AGROPECUÁRIA (Embrapa). Manual de métodos de análise de solo. Rio de Janeiro: Centro Nacional de Pesquisa de Solo, 1997.

FOLEGATTI, M. I. S. et al. Aproveitamento industrial do umbu: processamento de geleia e compota. Ciência e Agrotecnologia, Lavras, v. 27, n. 6, p. 1308-1314, 2003.

GOMES, J. M.; SILVA, A. R. Os substratos e sua influência na qualidade de mudas. In: BARBOSA, J. G. et al. (Eds.). Nutrição e adubação de plantas cultivadas em substrato. Viçosa: UFV, 2004. p. 190-225.
HARTMANN, H. T. et al. Plant propagation: principles and practices. 7. ed. New Jersey: Prentice Hall, 2002.

LIMA, E. D. P. A. et al. Caracterização física e química dos frutos da umbu-cajazeira (Spondias spp) em cinco estádios de maturação, da polpa congelada e néctar. Revista Brasileira de Fruticultura, Jaboticabal, v. 24, n. 2 , p. 338-343, 2002.

LIMA, J. D. et al. Efeitos da luminosidade no crescimento de mudas de Caesalpinia ferrea Mart. ex Tul. (Leguminosae, Caesalpinoideae). Acta Amazonica, Manaus, v. 38, n. 1, p. 5-10, 2008.

LIMA, J. F. et al. Avaliação de diferentes substratos na qualidade fisiológica de sementes de melão de caroá (Sicana odorifera (Vell.) Naudim). Revista Brasileira de Plantas Medicinais, Botucatu, v. 12, n. 2, p. 163-167, 2010.

LIMA, S. C. Germinação de sementes e otimização de técnicas de micropropagação de umbuzeiro (Spondias tuberosa) - Anacardiaceae. 2009. 96 f. Dissertação (Mestrado em Ciências Biológicas) - Universidade Federal do Rio Grande do Norte, Natal, 2009.

LOSS, A. et al. Indução do enraizamento em estacas de Malvaviscus arboreus Cav. com diferentes concentrações de ácido indolbutírico (AIB). Acta Scientiarum Agronomy, Maringá, v. 31, n. 2, p. 269-273, 2009.

MELO, A. P. C. de et al. Superação de dormência de sementes e crescimento inicial de plântulas de umbuzeiro. Semina: Ciências Agrárias, Londrina, v. 33, n. 4, p. 13431350, 2012.

NEVES, O. S. C. et al. Adição de níquel na solução nutritiva para o cultivo de mudas de umbuzeiro. Revista Brasileira de Ciências do Solo, Viçosa, v. 31, n. 3, p. 485490, 2007.

OHLAND, T. et al. Enraizamento de estacas apicais de figueira 'roxo de valinhos' em função de época de coleta e AIB. Ciência e Agrotecnologia, Lavras, v. 33, n. 1, p. 74-78, 2009.

OLIVEIRA, I. V. M. et al. Clonagem do abacateiro variedade "Duke 7" (Persea americana Mill.) por alporquia. Revista Brasileira de Fruticultura, Jaboticabal, v. 30, n. 3, p. 759-763, 2008 .

PIZZATTO, M. et al. Influência do uso de AIB, época de coleta e tamanho de estaca na propagação vegetativa de hibisco por estaquia. Revista Ceres, Viçosa, v. 58, n. 4, p. 487-492, 2011.

RIOS, E. S. et al. Concentrações de ácido indolbutírico, comprimento e época de coleta de estacas, na propagação de umbuzeiro. Revista Caatinga, Mossoró, v. 25, n. 1, p. 52-57, 2012. 
SMARSI, R. C. et al. Concentrações de ácido indolbutírico e tipos de substrato na propagação vegetativa de lichia. Revista Brasileira de Fruticultura, Jaboticabal, v. 30, n. 1, p. 7-11, 2008.

SOUZA, A. A. et al. Semillas de Spondias tuberosa oriundos de frutos cosechados en cuatro estadios de maturación y almacenadas. Revista Brasileira de Engenharia Agrícola e Ambiental, Campina Grande, v. 9, n. 3, p. 372-378, 2005.
SOUZA, E. P. Propagação da cajazeira e do umbuzeiro por meio de estaquia, alporquia e enxertia. 2007. $105 \mathrm{f}$. Dissertação (Mestrado em Agronomia) - Universidade Federal da Paraíba, Areia, 2007. 